\title{
Atom-probe Tomographic Study of Interfacial Intermixing and Segregation in InAs/GaSb Superlattices
}

Yifei Meng $^{1,2}$, Honggyu Kim ${ }^{1,2}$, Dieter Isheim ${ }^{3,4}$, David N. Seidman ${ }^{3,4}$, Jian-Min Zuo ${ }^{1,2}$

1. Dept of Materials Science and Engineering, University of Illinois, Urbana, Illinois, USA

2. Seitz Materials Research Laboratory, University of Illinois, Urbana, Illinois, USA

3. Dept of Materials Science and Engineering, Northwestern University, Evanston, Illinois, USA

4. Northwestern University Center for Atom-Probe Tomography (NUCAPT), Northwestern University, Illinois, USA

Mid-infrared detectors based on type-II InAs/GaSb superlattices (T2SLs), first proposed in the 1980s, have drawn increasing interest recently [1]. They overcome several problems inherent to traditional $\mathrm{HgCdTe}$ alloy detectors: specifically, (a) precision control of alloy composition, (b) non-uniformity over large areas and (c) large tunneling current [2]. The T2SLs are grown by molecular beam epitaxy (MBE). During the growth of T2SLs, composition deviation from the design and interfacial intermixing still limits, however, the device performance [3]. Recent developments in the technology of ultraviolet (UV) picosecond laser assisted local-electrode atom-probe (LEAP) tomography have made atom-probe tomography (APT) an important instrument for atomic concentration profiling with sub-nm spatial resolution [4].Therefore, we performed APT analyses to characterize the individual elemental concentration profiles in T2SLs.

We studied two InAs/GaSb superlattices grown by MBE on an AlSb capped GaSb substrate. Both T2SLs are based on alternating 7 GaSb monolayers (MLs) and 14 InAs MLs. InAs layers are compressively strained in the growth direction. Therefore, one of the two samples has an additional interfacial treatment, which consisted of depositing InSb at the InAs-on-GaSb interfaces to nullify the strain. No interfacial treatment was applied to the other sample. By comparing the two samples, we aim to understand how the interfacial treatment affects the concentration profiles and interface intermixing.

The APT samples were prepared using a dual beam focused ion beam (FIB) microscope. The APT experiment was performed employing a Cameca LEAP 4000X $\mathrm{Si}$ at Northwestern University, at a base temperature of $25 \mathrm{~K}$ and under ultra-high vacuum $\left(<8 \times 10^{-9} \mathrm{~Pa}\right)$. We utilized a focused UV picosecond laser to dissect the specimens on an atom-by-atom and atomic-plane by atomic-plane basis. To explore the influence of laser pulse energy on the accuracy of the APT results, we performed experiments with $5 \mathrm{pJ}$ per pulse and $1.4 \mathrm{pJ}$ per pulse laser pulse energy, at a $250 \mathrm{kHz}$ pulse repetition rate. The 3D APT data was reconstructed employing flat epitaxial layers (a known structural feature) as a reference standard. Additionally, we performed a density correction and rescaled in the z-direction to compensate for the deviation caused by the different evaporation fields of InAs and GaSb.

Figure 1(a) displays a 3D reconstruction of the atomic distribution of atoms in the $\mathrm{InAs} / \mathrm{GaSb}$ superlattice with interfacial InSb layers. While the 3D atom map clearly resolves the superlattice, quantitative measurements were performed utilizing the concentration profiles of each of the elements (Figure 1(b-e)). The results demonstrate that the GaSb-on-InAs interface is 
significantly sharper than the interface for the InAs-on-GaSb multilayer. It is noted that a higher level of intermixing occurs at the InAs-on-GaSb interface.

We also examined the concentration profiles in the form of $\mathrm{Ga} / \mathrm{Sb}$ and $\mathrm{In} / \mathrm{As}$ ratios. The ratio profiles exhibit dramatic changes at interfaces, which permits us to study segregation of $\mathrm{Ga}, \mathrm{In}$, and $\mathrm{Sb}$ in the MBE grown samples. For an improved understanding of interfacial segregation, we employ kinetic growth model to simulate the concentration profiles of all the elements. Preliminary results demonstrate that the simulations yield similar trends for interfacial atomic segregation as the APT data does.

In conclusion, individual concentration profiles were measured for InAs/GaSb T2SLs with subnanoscale spatial resolution. This APT permits a detailed comparison with the results of model kinetic growth simulations [5].

References:

[1] M. Z. Tidrow, Infrared Phys. Technol. 52 (2009), p. 322-325.

[2] D. R. Rhiger, J. Electron. Mater. 40 (2011), p. 1815-1822.

[3] J. Steinshnider et al, Phys. Rev. Lett. 85 (2000), p. 4562-4565.

[4] O. Moutanabbir et al, Appl. Phys. Lett. 98 (2011), p. 013111.

[5] This work is supported by U.S. Army Research Office (grant number Army W911NF-10-10524) through the MURI program. The LEAP tomograph at NUCAPT was purchased and upgraded with funding from NSF-MRI (DMR-0420532) and ONR-DURIP (N00014-0400798, N00014-0610539, N00014-0910781) grants. Instrumentation at NUCAPT was also supported by the Initiative for Sustainability and Energy at Northwestern (ISEN).

(a)

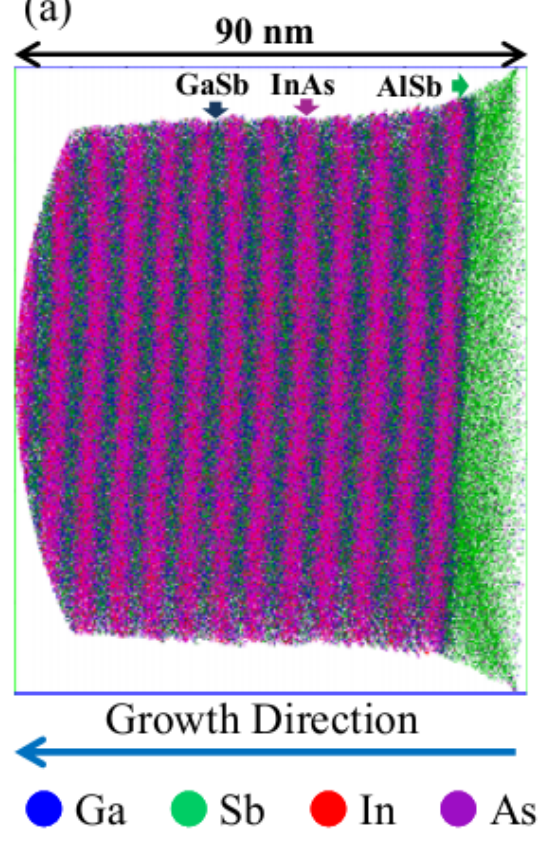

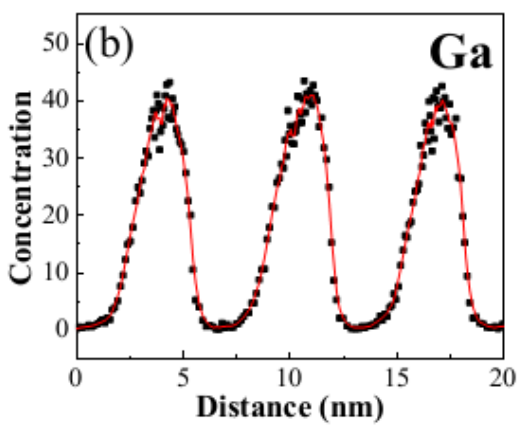
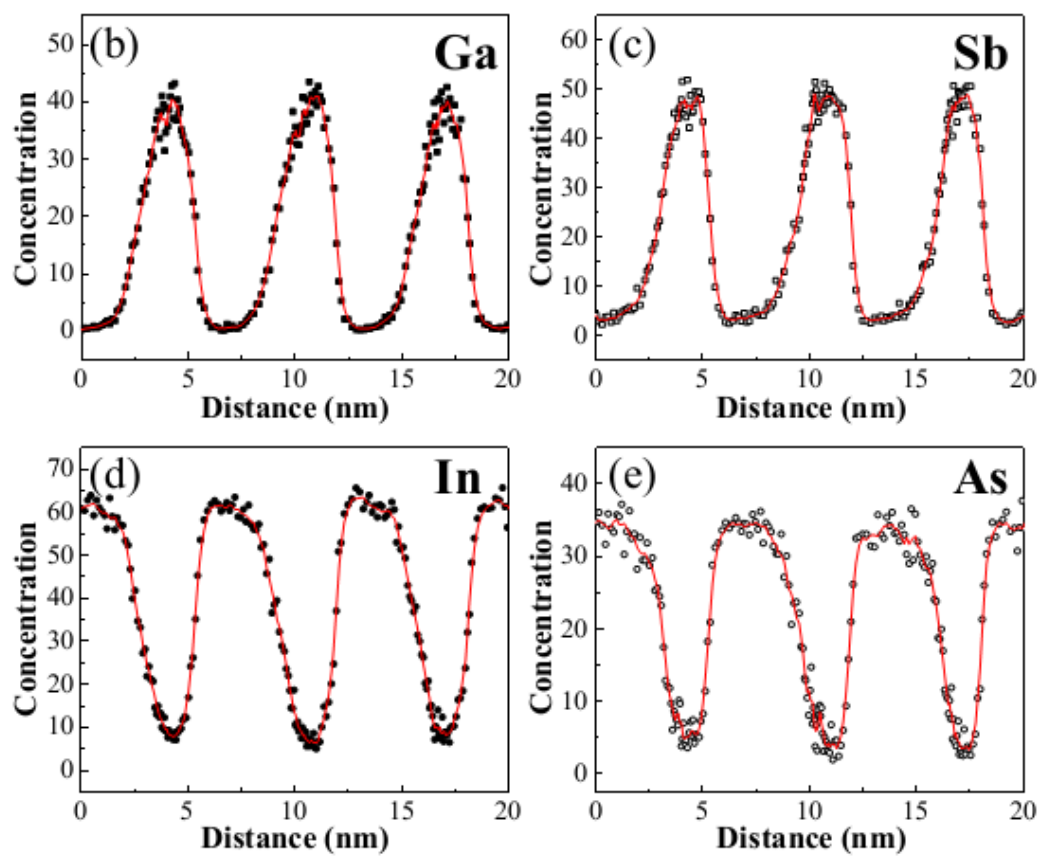

Figure 1. (a) Atom-by-atom 3D APT reconstruction of InAs/GaSb superlattice near the AlSbcapped GaSb substrate region. Concentration profiles along the growth direction for: (b) $\mathrm{Ga}$; (c) $\mathrm{Sb}$; (d) In; and (e) As. 